\title{
A mutation in the low voltage-gated calcium channel CACNA1G alters the physiological properties of the channel, causing spinocerebellar ataxia
}

Hiroyuki Morino ${ }^{1 *}$, Yukiko Matsuda', Keiko Muguruma², Ryosuke Miyamoto', Ryosuke Ohsawa', Toshiyuki Ohtake ${ }^{3}$, Reiko Otobe ${ }^{4}$, Masahiko Watanabe ${ }^{5}$, Hirofumi Maruyama ${ }^{6}$, Kouichi Hashimoto $^{7}$ and Hideshi Kawakami ${ }^{*}$

\begin{abstract}
Background: Spinocerebellar ataxia (SCA) is a genetically heterogeneous disease. To date, 36 dominantly inherited loci have been reported, and 31 causative genes have been identified.

Results: In this study, we analyzed a Japanese family with autosomal dominant SCA using linkage analysis and exome sequencing, and identified CACNA1G, which encodes the calcium channel Cav3.1, as a new causative gene. The same mutation was also found in another family with SCA. Although most patients exhibited the pure form of cerebellar ataxia, two patients showed prominent resting tremor in addition to ataxia. Cav3.1 is classified as a lowthreshold voltage-dependent calcium channel (T-type) and is expressed abundantly in the central nervous system, including the cerebellum. The mutation p.Arg1715 His, identified in this study, was found to be located at S4 of repeat IV, the voltage sensor of the Cav3.1. Electrophysiological analyses revealed that the membrane potential dependency of the mutant Cav3.1 transfected into HEK293T cells shifted toward a positive potential. We established induced pluripotent stem cells (iPSCs) from fibroblasts of the patient, and to our knowledge, this is the first report of successful differentiation from the patient-derived iPSCs into Purkinje cells. There was no significant difference in the differentiation status between control- and patient-derived iPSCs.
\end{abstract}

Conclusions: To date, several channel genes have been reported as causative genes for SCA. Our findings provide important insights into the pathogenesis of SCA as a channelopathy.

Keywords: Spinocerebellar ataxia, CACNA1G, T-type calcium channel, Cav3.1, Induced pluripotent stem cell

\section{Background}

Spinocerebellar ataxia (SCA) is characterized by cerebellar ataxia, dysarthria, oculomotor disorder, extrapyramidal sign, motor neuron sign, epilepsy, cognitive impairment, and many other symptoms [1]. To date, 36 loci have been shown to be involved in autosomal dominant SCA, and the causative genes and mutations have been identified for 31 types of SCAs [2]. The majority of SCAs account for repeat expansion, including a coding CAG repeat (SCA1$3,6,7,17$, and dentatorubral-pallidoluysian atrophy)

\footnotetext{
* Correspondence: morino@hiroshima-u.ac.jp; hkawakam@hiroshima-u.ac.jp ${ }^{1}$ Department of Epidemiology, Research Institute for Radiation Biology and Medicine, Hiroshima University, Hiroshima, 1-2-3, Kasumi, Minami-ku, Hiroshima 734-8553, Japan

Full list of author information is available at the end of the article
}

causing misfolding of the encoded protein due to the expanded polyglutamine tracts; groups of noncoding CAG, CTG, ATTCT, and GGCCTG repeats (SCA8, 10, 12, and 36); and large TGGAA repeat insertions (SCA31) that induce RNA-mediated gain-of-function mechanisms. The remaining known SCA types are caused by single nucleotide variants or indels (SCA5, 11, 13-15, 18-21, 23, 26$28,34,35,38,40$, and 41 ) in genes that encode various functional proteins [2].

CACNA1A (MIM 601011) was first reported as a causative gene of SCA6 (MIM 183086) among the genes encoding channels $[3,4]$. In recent years, potassium channel mutations have been described in SCA13 (MIM 605259) and SCA19/22 (MIM 607346) [5-7]. Episodic 
ataxia (EA) is one of the diseases associated with SCA, and some types of EA are caused by mutations in calcium channel. CACNA1A and CACNB4 mutations lead to EA2 (MIM 108500) and EA5 (MIM 613855), respectively $[8,9]$. Thus the ion channel dysfunction plays a key role in the pathogenesis of ataxia and related diseases.

We have been engaged in clinicogenetic research using the samples from more than 2,000 patients with SCA. From a thorough examination of the disease types and the geographical distributions of the 2,121 patients, causative genes for $26.6 \%$ of the dominant-inherited cases (205 out of 721) are still unknown [10]. Therefore, in order to identify novel causative genes, we applied exome sequencing to the families with dominantly inherited SCA.

\section{Methods}

\section{Patients}

We enrolled two Japanese families with segregating dominant traits for cerebellar ataxia. There are 10 affected individuals in the family 1 and five in the family 2 . Blood samples were obtained from eight affected individuals and three unaffected individuals in family 1 , and five affected individuals and two unaffected individuals in family 2 (Fig. 1a). All patients were diagnosed with SCA by neurologists. Prior to this study, we confirmed that all affected individuals had no pathogenic mutations causing SCA1$3,6,8$, and dentatorubral-pallidoluysian atrophy. The study was approved by the Human Subjects Committees of Hiroshima University; all subjects provided written informed consent.

\section{Linkage analysis}

The samples used for linkage analysis were 1-III-2, 1-III4, 1-III-6, 1-III-8, 1-III-11, 1-III-13, 1-IV-2, 1-IV-3, and 1-IV-4. Because it was possible that 1-IV-3 and 1-IV-4 did not reach the appropriate age at onset, the two samples were treated as unknown in the pedigree file of linkage analysis. Genomic DNA (gDNA) was extracted from the peripheral lymphocytes of the participants according to standard protocols. We used a Genome-Wide Human SNP Array 6.0 (Affymetrix, Santa Clara, CA, USA) for genotyping of single nucleotide polymorphisms (SNPs), and linkage analysis was performed by Allegro software, estimating the dominant inheritance [11].

\section{Exome Sequencing}

Exome sequencing was carried out using three samples from 1-III-6, 1-III-11, and 1-III-13, as previously described [12]. For family 2, exome sequencing was also performed with the sample from 2-III-1. gDNA libraries were prepared using a SeqCap EZ Human Exome Library v2.0 (Roche, Basel, Switzerland). Sequencing was performed with 100-bp paired-end reads on a HiSeq2000 sequencer (Illumina, San Diego, CA, USA). We used BWA (http://bio-bwa.sourceforge.net/) [13] for alignment and mapping, Samtools (http://samtools.sourceforge.net/) [14] and Picard (http://broadinstitute.github.io/picard/) for SAM/BAM handling, GATK (http://www.broadinstitute.org/gatk/) [15] and Samtools for variant calls, and Annovar (http://annovar.openbioinformatics.org/) [16] for annotation. Functional predictions due to amino acid changes were estimated using PolyPhen-2 (http:// genetics.bwh.harvard.edu/pph2/) [17], SIFT (http://sift. bii.a-star.edu.sg/) [18], and Mutation Taster (http:// www.mutationtaster.org/index.html) [19]. Control exome sequences were obtained from Japanese patients undergoing exome analysis for diseases other than SCA. All reported genomic coordinates were in GRCh37/hg19. The identified mutations were validated with a standard polymerase chain reaction (PCR)based amplification followed by sequence analysis with an Applied Biosystems 3130 DNA sequencer (Thermo Fisher Scientific, Waltham, MA, USA).

\section{Expression vector}

Wild-type CACNA1G (short isoform; BC110995.1, NM_198382.2) in the pCMV-SPORT6 plasmid (pCMVSPORT6-CACNA1G) was purchased from Dharmacon (Lafayette, CO, USA). The mutation c.5075G > A corresponding to c.5144G $>\mathrm{A}$ in the longest isoform (NM_018896.4) was introduced by site-directed mutagenesis using QuikChange Lightning (Agilent Technologies, Santa Clara, CA, USA) and verified by bidirectional sequencing. The IRES-EGFP sequence was amplified by PCR from the pIRES-EGFP plasmid and inserted at the termination codon of the CDNA sequence in PCMV-SPORT6CACNA1G (pCMV-SPORT6-CACNA1G-IG) using an In-Fusion HD Cloning Kit (TaKaRa Bio, Shiga, Japan).

\section{Cell culture, transfection, and immunofluorescence}

The primary antibodies used in this study were antiCACNA1G [20], and anti-alpha 1 sodium potassium ATPase (Abcam, Cambridge, UK).

HeLa and HEK293T cells were maintained in Dulbecco's modified Eagle's medium (DMEM; Nakarai Tesque, Kyoto, Japan) supplemented with $10 \%$ fetal bovine serum and penicillin/streptomycin (PS) in a $37{ }^{\circ} \mathrm{C}$ incubator with $5 \% \mathrm{CO}_{2}$. For immunofluorescence analysis, cells were grown on chamber slides (SCS-008; Matsunami, Osaka, Japan) coated with poly-L-lysine (SigmaAldrich, St. Louis, MO, USA), and were transiently transfected with pCMV-SPORT6-CACNA1G using Lipofectamine LTX (Thermo Fisher Scientific, Waltham, MA, USA), according to the manufacturer's instructions. After $48-72 \mathrm{~h}$, the cells were fixed in $4 \%$ paraformaldehyde, washed with phosphate-buffered saline (PBS), blocked, and permeabilized with $0.2 \%$ Tween 20 . Cells 

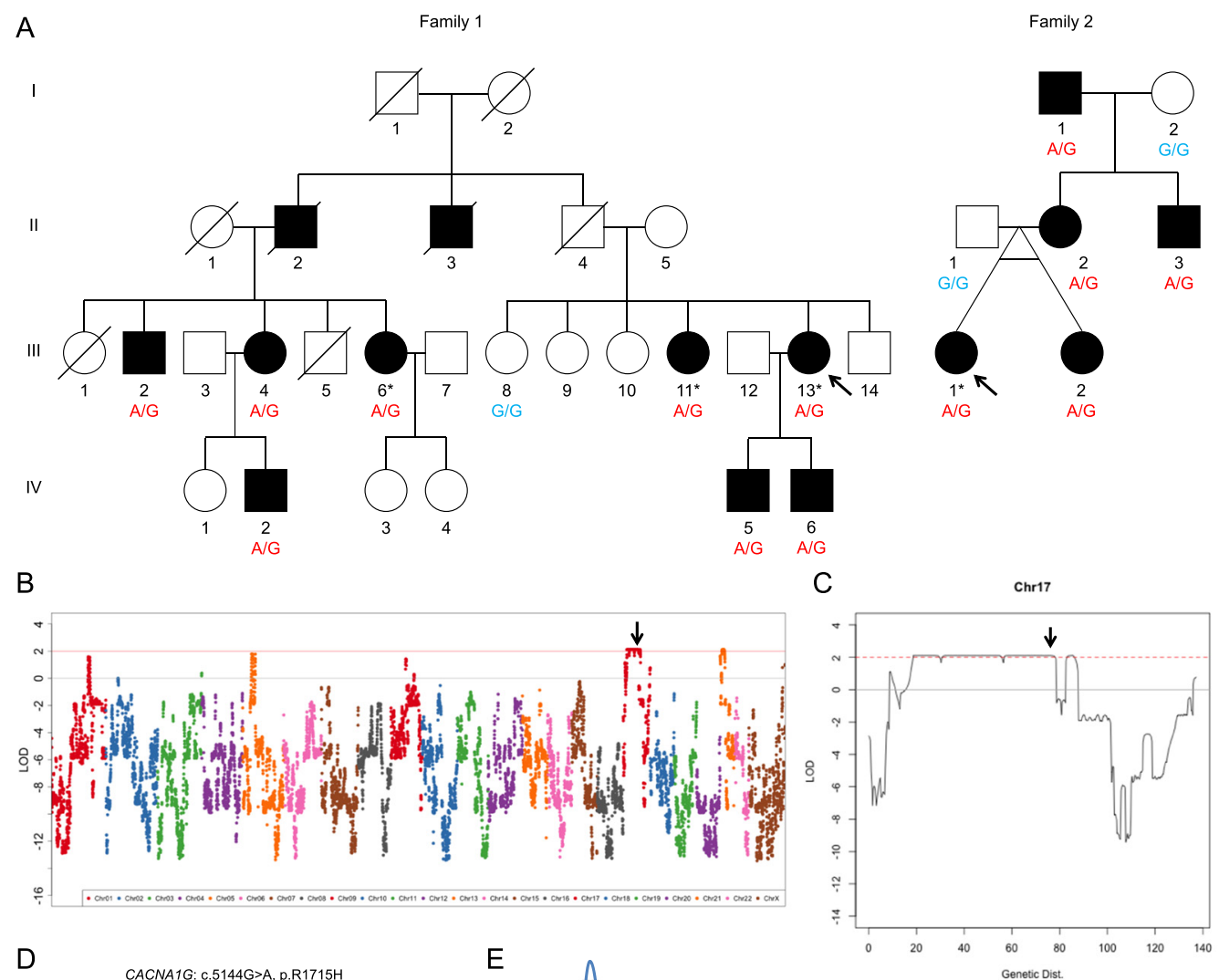

D $\frac{\text { Arg }}{A \text { G G G T G }} \frac{\text { Val }}{C \text { T T G }} \frac{\text { Arg//is }}{C G / A C} \frac{\text { lle }}{\text { A T T }} \frac{\text { Ala }}{\text { G C C }} \frac{\text { Arg }}{C \text { G A }}$ Mutant

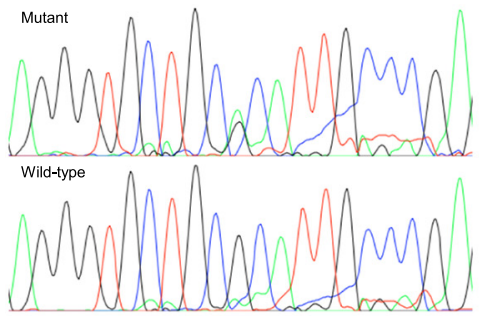

$\mathrm{F}$

<Genomic sequence> Homo sapiens Gorilla gorilla Macaca mulatta

Oryctolagus cuniculus Mus musculus

Gallus gallus Xenopus tropicalis Takifugu rubripes

<Protein sequence> Homo sapiens

Gorilla gorilla

Macaca mulatta

oryctolagus cuniculus

Mus musculus

Gallus gallus

Xenopus tropicalis

Takifugu rubripes

Fig. 1 (See legend on next page.)

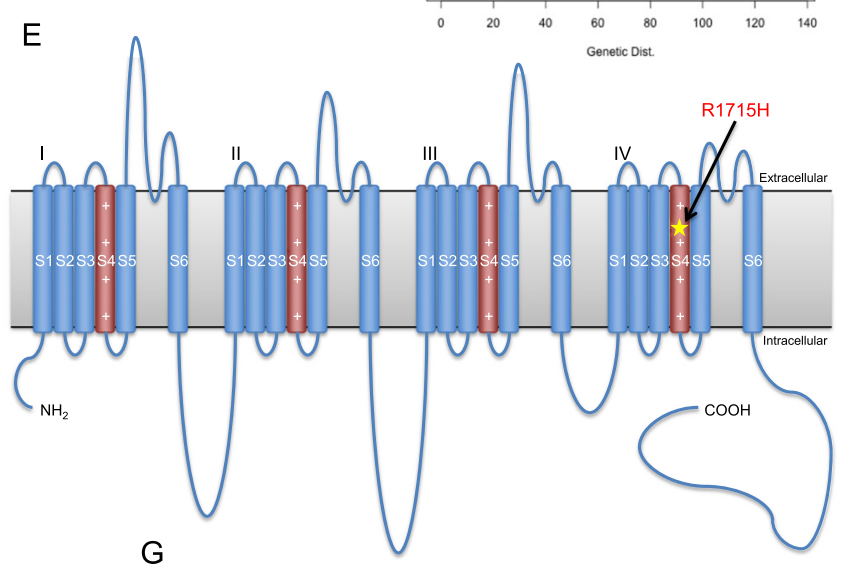

Common: $48,372,363-48,735,774360 \mathrm{~kb}$ Family 1: $42,085,972-52,097,650$ 10Mb

AGGGTGCTGCGCATTGCCCGA AGGGTNCTGCGCATTGCCCGA AGGGTACTGCGCATTGCCCGA AGGGTGCTGCGCATCGCCCGA AGGGTGCTCCGCATTGCTCGA AGGGTTCTCAGGATTGCTCGA AGGGTCCTGCGGATCGCTCGC AGGGTGCTGAGAATCGCCCGA

PTI IRIMRVLRIARVLKLLKM PTIIRIMRVLRIARVLKLLKM PTIIRIMRVLRIARVLKLLKM PTIIRIMRVLRIARVLKLLKM PT I IRIMRVLRIARVLKLLKM PTI IRIMRVLRIARVLKLLKM PTIIRIMRVLRIARVLKLLKM PTIIRIMRVLRIARVLKLLKM
Family 2: $38,277,468-57,466,53119 \mathrm{Mb}$

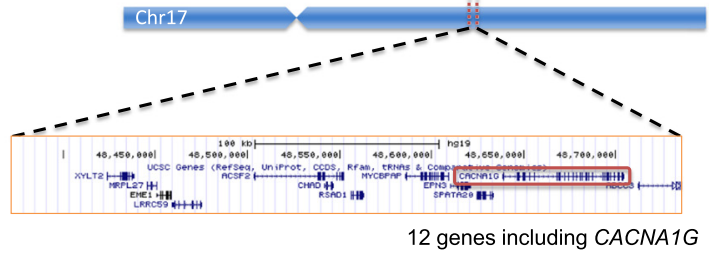


(See figure on previous page.)

Fig. 1 Identification of a mutation in CACNA1G causing SCA. a Pedigree charts of families 1 and 2. Arrows indicate the probands. Filled and open symbols represent affected and unaffected individuals, respectively. Genotypes of the variant c.5144G $>$ A are shown under the number of samples. Asterisks indicate the patients used for exome sequencing. $\mathbf{b}, \mathbf{c}$ Results of linkage analysis. Arrows indicate the positions of CACNA1G. $\mathbf{d}$ Sanger sequencing to confirm the CACNA1G variant. The reference nucleotide $\mathrm{G}$ is overlapped with variant nucleotide $\mathrm{A}$ in the mutant sample. $\mathbf{e}$ Structure of Cav3.1 encoded by CACNA1G. The star indicates the position of the identified mutation. The mutation was located in the segment 4 (S4) of the fourth repeat. $\mathbf{f}$ Conservation at the location of the mutation. The nucleotide and amino acid sequences are completely conserved among vertebrates. $\mathbf{g}$ Haplotype analysis. From the result of SNP genotyping, the haplotypes of both families around the CACNA1G gene coincided for $360 \mathrm{~kb}$

were incubated overnight at $4{ }^{\circ} \mathrm{C}$ with anti-CACNA1G and anti-alpha 1 sodium potassium ATPase antibodies and then treated with secondary antibodies. Images were obtained using confocal microscopy (LSM510; Carl Zeiss, Jena, Germany). The nuclei were visualized using DAPI.

Cells for whole-cell patch clamping were grown in glass-bottom plates ( $\mu$-Dish $35 \mathrm{~mm}$ low; ibidi, Martinsried, Germany) for $24 \mathrm{~h}$ following transfection with SPORT6-CACNA1G-IG using Lipofectamine LTX.

\section{Electrophysiology}

Whole-cell recordings were made from GFP-expressing HEK293T cells using an upright microscope (BX51WI; Olympus, Tokyo, Japan) equipped with an IR-CCD camera system (IR-1000; DAGE-MTI, Michigan, IN, USA) at room temperature. The intracellular solution was composed of $110 \mathrm{mM} \mathrm{CsCl}, 20 \mathrm{mM}$ TEA-Cl, $10 \mathrm{mM} \mathrm{NaCl}$, $5 \mathrm{mM}$ EGTA, $10 \mathrm{mM}$ HEPES, $4 \mathrm{mM} \mathrm{Mg}$-ATP, and $0.4 \mathrm{mM} 2 \mathrm{Na}-\mathrm{GTP}$ ( $\mathrm{pH} 7.3$, adjusted with $\mathrm{CsOH}$ ). The pipette access resistance was about 2-3 M $\Omega$. The composition of the extracellular solution was $10 \mathrm{mM} \mathrm{NaCl}$, $105 \mathrm{mM}$ TEA-Cl, $10 \mathrm{mM}$ 4-AP, $2.5 \mathrm{mM} \mathrm{KCl}, 2 \mathrm{mM}$ $\mathrm{CaCl}_{2}, 1 \mathrm{mM} \mathrm{MgSO}_{4}, 1.25 \mathrm{mM} \mathrm{NaH} \mathrm{PO}_{4}, 26 \mathrm{mM}$ $\mathrm{NaHCO}_{3}$, and $20 \mathrm{mM}$ glucose, bubbled with $95 \% \mathrm{O}_{2}$ and $5 \% \mathrm{CO}_{2}$. Ionic currents were recorded with an EPC-10 (HEKA Elektronik, Lambrecht, Germany). The signals were filtered at $3 \mathrm{kHz}$ and digitized at $20 \mathrm{kHz}$. On-line data acquisition and off-line data analysis were performed using PATCHMASTER software (HEKA Elektronik, Lambrecht, Germany). Relative conductance and steady-state inactivation plots were fitted by the following Boltzmann equations:

$$
\begin{aligned}
\frac{G}{G_{\text {max }}} & =\frac{1}{1+\exp \left(\left(V_{\text {half }}-V_{m}\right) / k\right)} \\
\frac{I}{I_{\text {max }}} & =\frac{1}{1+\exp \left(\left(V_{m}-V_{\text {half }}\right) / k\right)}
\end{aligned}
$$

\section{Derivation of patient-specific fibroblasts and generation} of induced pluripotent stem cells (iPSCs)

Patient 2-III-1 harbored the CACNA1G mutation. Thus, a 3-mm punched skin biopsy was obtained from the upper arm. The skin sample was mechanically cut into small pieces and cultured in 100-mm cell culture dishes in DMEM containing $20 \%$ fetal bovine serum. Outgrowth of dermal fibroblasts appeared after 7-14 days, and cultures were split at 1:3 after reaching $80 \%$ confluence. Peripheral blood was obtained from a healthy donor after providing written informed consent in accordance with the institutional review board guidelines. Peripheral blood mononuclear cells (PBMCs) were purified by density gradient centrifugation with a BD Vacutainer CPT (BD Biosciences, Franklin Lakes, NJ, USA) according to the manufacturer's instructions.

Human complementary DNAs for reprogramming factors were transduced into dermal fibroblasts and PBMCs with episomal vectors (pCXLE-hOCT3/4-shp53-F, pCXLEhSK, and pCXLE-hUL; Addgene, Cambridge, MA, USA) $[21,22]$. The generated iPSCs were maintained on a mitomycin C-inactivated SNL feeder cell layer in DMEM/F12 containing GlutaMaxI (Thermo Fisher Scientific, Waltham, MA, USA) supplemented with 20 \% KnockOut Serum Replacement (KSR; Thermo Fisher Scientific, Waltham, MA, USA), $5 \mathrm{ng} / \mathrm{ml}$ recombinant human FGF basic (Wako, Osaka, Japan), $0.1 \mathrm{mM} \beta$-mercaptoethanol (Sigma-Aldrich, St. Louis, MO, USA) and PS under $5 \% \mathrm{CO}_{2}$.

\section{Purkinje cell differentiation from iPSCs}

The differentiation of Purkinje cells from iPSCs was performed as described previously [23, 24].

\section{Results}

\section{Clinical manifestations}

The proband 1-III-13 has exhibited the pure form of cerebellar ataxia, and her age of at disease onset was 45 years. The main symptoms were ataxic gait, dysarthria, and gaze-evoked horizontal nystagmus. Cognitive impairment, epilepsy, muscle atrophy, involuntary movement, and parkinsonism were not observed. The parents did not exhibit the same symptoms; however, symptoms were observed in her uncles. Her sister (1-III-11) also suffered from the same symptoms, with slightly increased severity. Other affected members in the family 1 showed similar clinical features, and the age at onset varied from 20 to 70 years. In all patients, progression of the disease was relatively slow. 
For family 2, the age at onset ranged from 18 to 57 years. The main symptoms and clinical course were similar to those in family 1 , except that two patients 2II-2 and 2-III-2 showed prominent resting tremors.

In neuroimaging, brain magnetic resonance imaging (MRI) results of 2-III-1 demonstrated cerebellar atrophy, typical of SCA. However, the cerebrum and brainstem showed no abnormalities (Additional file 1: Figure S1).

\section{Candidate region from linkage analysis}

From the results of linkage analysis, two regions where logarithm of odds (LOD) score was 2 or more were obtained in chromosomes 17 and 21 (Fig. 1b). The maximum LOD scores for chromosomes 17 and 21 were 2.1067 and 2.1066, respectively. The candidate region on chromosome 17 was relatively wide, approximately $60 \mathrm{cM}$ (Fig. 1c). The region did not correspond with any genetic loci of known SCAs.

\section{Variant filtration}

The results of exome sequencing are shown in Additional file 2: Table S1. More than 90,000 variants in each sample were called. We narrowed down the candidate variants based on filtering criteria consisting of open databases (dbSNP, 1000 Genomes, and EPS 6400), genomic position, function, and zygosity. A total of 386 variants were common to 1-III-6, 1-III-11, and 1-III-13. Among them, we excluded variants that were observed in other diseases from our in-house variant database, and 11 heterozygous variants remained (Additional file 3: Table S2).

Variants that were estimated to be pathogenic by two or more of the three prediction algorithms were as follows: SLC15A1, ALOX15B, CACNA1G, and RNF213. We performed segregation of the variants with the disease in the 11 available subjects from family 1 . The variants in $A L O X 15 B$ and $C A C N A 1 G$ were observed only in affected subjects, but not in unaffected subjects. Based on the linkage analysis, the variant of CACNA1G was present in the high LOD region; therefore, we concluded that CACNA1G was the most likely candidate. This variant was confirmed by Sanger sequencing (Fig. 1d).

\section{Heterozygous mutation in CACNA1G}

Chr17:48694921G > A corresponded to CACNA1G:c. 5144G > A, p.Arg1715His (NM_018896.4, MIM 604065) (Fig. 1d). The CACNA1G gene encodes the Cav3.1 T-type voltage-dependent calcium channel (VDCC). The nucleotide and amino acid sequences of the mutated region were found to be completely conserved among vertebrates (Fig. 1f). The structure of the calcium channel $\alpha$ subunit, including $\mathrm{Ca}_{\mathrm{V}} 3.1$, consisted of four repeating domains (repeats I-IV) comprised of five hydrophobic segments (S1-3, S5, and S6) and one positively charged segment (S4) each [25]. The identified mutation was located in S4 of repeat IV (Fig. 1e).

Identical heterozygous mutation was found in the variants obtained by exome sequencing for the sample from 2-III-1 of family 2. No other variants that caused SCA were found in this patient. Sequencing of CACNA1G throughout family 2 revealed that affected members harbored the mutation and unaffected members did not. From the results of SNP genotyping performed for linkage analysis, we compared the haplotypes around the CACNA1G gene in the two families. Families 1 and 2 shared the haplotypes in the range of 10 and $19 \mathrm{Mb}$, respectively. Both families shared the haplotypes for a length of $360 \mathrm{~kb}$ (Fig. 1g).

\section{Localization of mutant $\mathrm{Ca}_{\mathrm{v}} 3.1$}

Immunocytochemical studies were performed in order to examine the possible changes in the subcellular localization caused by the mutation. We used the NaKATPase as a plasma membrane marker. Both wild-type and mutant $\mathrm{Ca}_{\mathrm{v}} 3.1$ were distributed diffusely in the cytoplasm and the plasma membrane (Additional file 4: Figure S2). No significant differences were observed in the localizations of wild-type and mutant $\mathrm{Ca}_{\mathrm{V}} 3.1$.

\section{Electrophysiological properties}

We examined changes in the electrophysiological properties of mutant $\mathrm{Ca}_{\mathrm{V}} 3.1$. Whole-cell recording was obtained from GFP-positive HEK293T cells expressing wild-type or mutant $\mathrm{Ca}_{\mathrm{V}}$ 3.1. To isolate $\mathrm{Ca}^{2+}$ currents, HEK293T cells were bathed with the extracellular solution containing potassium channel blockers.

T-type VDCC currents were elicited by depolarizing voltage steps after a hyperpolarizing prepulse to $-100 \mathrm{mV}$ (300 ms) in HEK293T cells carrying wild-type and mutant constructs (Fig. 2a). The peak current amplitudes are plotted against step voltages in Fig. 2b. In HEK293T cells expressing the mutant construct, amplitudes of T-type VDCC currents were significantly smaller at step voltages of -60 and $-70 \mathrm{mV}$, but not at higher membrane potential depolarizations (Fig. 2a). This result suggested that the maximum conductance was normal, but that activation of mutant $\mathrm{Cav}_{\mathrm{v}} 3.1$ was shifted to depolarized membrane potentials. To further examine this phenomenon, the, estimated relative conductances were calculated from the peak current-voltage relationships. The reversal potentials used for correction were estimated by line fits of peak amplitudes from -10 to $+30 \mathrm{mV}$. For each cell, the relative conductance was plotted against depolarizing voltage pulses and fitted by a Boltzmann equation (Fig. 2c). The averaged half-conductance potential of $-57.5 \pm 9.1 \mathrm{mV}$ in wild-type $(\mathrm{n}=9$, mean $\pm \mathrm{SD})$ was more hyperpolarized than that of $-44.7 \pm 6.0 \mathrm{mV}$ in mutant $(\mathrm{n}=12 ; p=0.004$, Mann-Whitney $U$-test), suggesting that activation of 
A

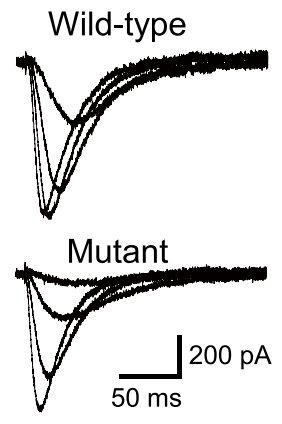

C

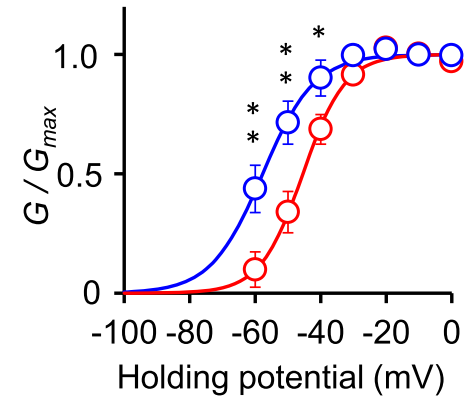

E

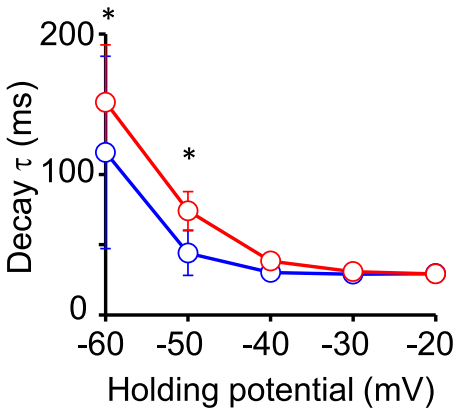

B

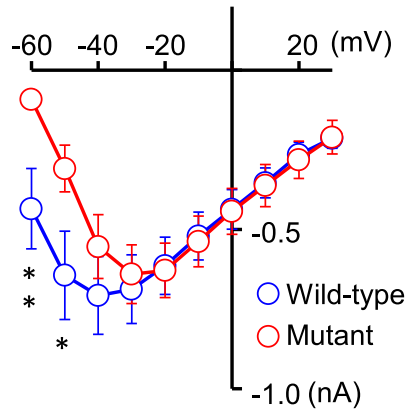

D

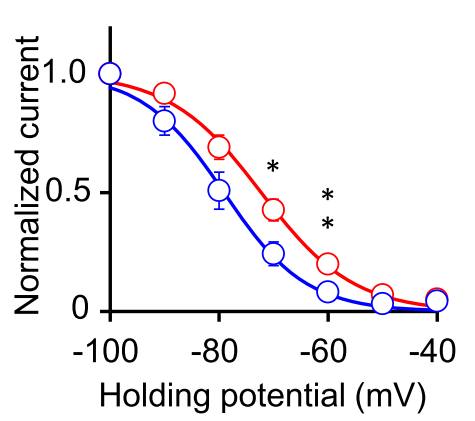

F

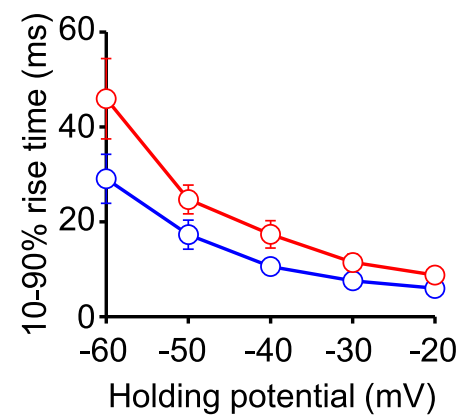

Fig. 2 Changes in electrophysiological properties. a Representative traces of T-type VDCC currents recorded from HEK293T cells expressing wild-type (upper) or mutant (lower) Cav3.1. Holding potential was -60 mV. Voltage steps were applied after a hyperpolarizing prepulse to -100 mV (duration $=500$ ms). b Peak current-voltage plots of VDCC currents of wild-type (blue, $n=9)$ and mutant (red, $n=12$ ) Cav3.1. c Relative conductance-voltage plots. Each data point was calculated from data in B. The black line is a fit using the Boltzmann equation (see Methods). d Steady state inactivation-voltage plots. The voltage step to $-30 \mathrm{mV}$ was preceded by incremental hyperpolarizing pulses (duration = $300 \mathrm{~ms}$ ). Data were obtained from the same cells shown in B and C. The black line is a fit using the Boltzmann equation (see Methods). e The decay time constants of inactivation of the $\mathrm{Ca}^{2+}$ current plotted against voltage steps. $\mathbf{f}$ The $10-90 \%$ rise times of Ca ${ }^{2+}$ currents plotted against voltage steps. Data are presented as the mean \pm SEM. Statistical significance was assessed by Mann-Whitney $U$-test. ${ }^{* *} p<0.01,{ }^{*} p<0.05$

mutant $\mathrm{Ca}_{\mathrm{V}} 3.1$ was significantly shifted toward more positive membrane potentials. In contrast, the slope factor was not significantly different (wild-type, $6.4 \pm 2.2 \mathrm{mV}$; mutant, $6.2 \pm 2.9 \mathrm{mV} ; p=0.303$ ), suggesting parallel shift of membrane potential dependent curve to positive potentials. Steady-state inactivation was calculated by fitting the relative amplitudes of the currents as a function of the hyperpolarizing prepulse with the Boltzmann equation (Fig. 2d). Similar to activation, the half-steady-state inactivation potentials were significantly shifted to more positive membrane potentials (wild-type, -79.1 $\pm 6.6 \mathrm{mV}$; mutant, $-72.5 \pm 5.5 \mathrm{mV} ; p<0.025)$, but the slope factor was not significantly different (wild-type, $6.3 \pm 1.4 \mathrm{mV}$; mutant, $7.7 \pm 1.5 \mathrm{mV} ; p=0.07)$. As a consequence, the window current shifted toward more positive membrane potentials. The kinetics of the T-type VDCC current were also affected. The inactivation decay time constant of the T-type VDCC currents was significantly slower at small voltage steps in HEK293T cells expressing the mutant construct (Fig. 2e). While it was not statistically significant, a similar trend was also observed for the 10-90\% rise time (Fig. 2f). These lines of evidence suggested that the mutation affected the voltage dependency of activation and inactivation of the $\mathrm{Ca}_{\mathrm{v}} 3.1$ T-type VDCCs. 


\section{Purkinje cells derived from iPSCs}

We previously reported that the cerebellar neuroepithelium self-organizes in 3D human embryonic stem cell culture. The self-organized cerebellar neuroepithelium differentiates into mature Purkinje cells [24]. To examine the ability of the generated iPSCs to differentiate into Purkinje cells, we applied the 3D system to iPSC culture. The differentiated iPSC-derived cells expressed L7. The $\mathrm{L}^{+}$cells developed elaborate dendritic branches and spines that were positive for Purkinje cell-specific glutamate receptor GRID2 (Fig. 3, total culture days 51-58). From the result of morphological evaluation of differentiated Purkinje cells, dendritic field areas were 14,367 \pm $1,026 \mu \mathrm{m}^{2}$ in control $(\mathrm{n}=6$, mean \pm SD) and 12,287 \pm $954.8 \mu^{2}$ in patient-derived iPSC $(n=6$, mean \pm SD; Additional file 5: Figure S3A), and diameters of cell soma were $29.8 \pm 0.55 \mu \mathrm{m}$ in control $(\mathrm{n}=7$, mean $\pm \mathrm{SD})$ and $30.09 \pm 2.22 \mu \mathrm{m}$ in patient-derived $(\mathrm{n}=7$, mean $\pm \mathrm{SD}$; Additional file 5: Figure S3B). There were no obvious differences in the differentiation potentials between controland patient-derived neurons immunocytochemically and morphologically.

\section{Discussion}

Our study revealed that a mutation in CACNA1G encoding the T-type calcium channel $\mathrm{Ca}_{\mathrm{V}} 3.1$ causes SCA. This finding is consistent with the fact that several mutations in channel-encoding genes have been reported to cause SCA. We identified the mutation from a large Japanese family with autosomal dominant SCA using

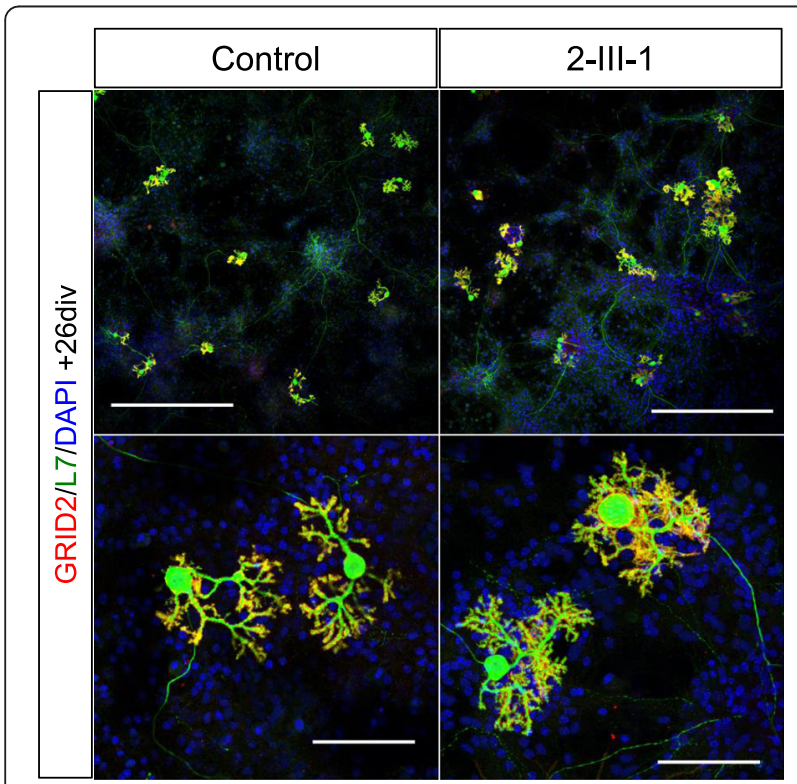

Fig. 3 iPSC-derived Purkinje cells. Immunostaining of iPSC-derived Purkinje cells on co-culture days 26. GRID2 was expressed in the $\mathrm{L}^{+}$ dendritic spines. The scale bars represent $500 \mu \mathrm{m}$ (upper panels) and $100 \mu \mathrm{m}$ (lower panels) exome sequencing and linkage analysis. Moreover, the same mutation was found in another Japanese family with SCA.

The mutation was located in the S4 segment, which is assumed as a part of the voltage sensor. In particular, the substituted arginine is one of the positively charged residues found every three amino acids; these residues play a central role in voltage sensing. Since simultaneous activation of all four voltage sensors is required to open the calcium channel, the arginine residue mutated in the affected individuals is electrophysiologically important [26]. Our electrophysiological analysis supported this concept. While the subcellular distribution and membrane translocation of the protein were normal, membrane potential dependency of activation and inactivation curves of $\mathrm{Ca}^{2+}$ currents were positively shifted in HEK293T cells expressing mutant $\mathrm{Ca}_{\mathrm{v}} 3.1$. Very recently, identical mutation in CACNA1G was reported in French families, which also demonstrated positive shifts in the window current of the T-type channel [27]. Alterations of T-type $\mathrm{Ca}^{2+}$ currents were consistent with our results. The mild physiological changes could explain the clinical features of SCA, including the age at onset and slow progression of the disease.

$\mathrm{Ca}_{\mathrm{V}} 3.1$ is expressed in the cerebellum, inferior olive nucleus, and the thalamus [28]. Physiologically, the channel is associated with high-frequency burst firing of more than $10 \mathrm{~Hz}$ and oscillation $[29,30]$ and regulates the extension of the axons and dendrites in the developmental stage [31]. In animal models, $\mathrm{Ca}_{V} 3.1$ has been implicated in epilepsy. $\mathrm{Ca}_{\mathrm{V}} 3.1$ knockout mice are unable to elicit thalamocortical low-threshold calcium potentials (LTCPs) and burst firing in response to hyperpolarization and are resistant to drug-induced absence seizures [32]. Conversely, overexpression of $\mathrm{Ca}_{\mathrm{V}} 3.1$ in transgenic mice induces thalamocortical spike-wave oscillations characteristic of pure absence epilepsy [33]. LTCPs of the thalamus are also important for stabilizing NREM sleep [34]. In another study using $\mathrm{Ca}_{\mathrm{V}} 3.1$ knockout mice, $\mathrm{Ca}_{\mathrm{V}} 3.1$ expressed in the inferior olive was shown to be associated with tremor [30].

The clinical picture of our patients was similar to that of SCA6, caused by mutation in another type of calcium channel, CACNA1A. Most patients exhibited the pure form of cerebellar ataxia. Notably, two patients had resting tremors. $\mathrm{Ca}_{\mathrm{V}} 3.1$ is expressed at high levels in the cerebellum and inferior olive nucleus. Because T-type VDCCs have important roles in the rhythmogenesis of inferior olivery neurons [35], the symptom may also be the consequence of the $\mathrm{Ca}_{\mathrm{V}} 3.1$ mutation. Pyramidal tract signs, which are characteristic of the French families, were not prominent in our cases [27]. The fact that the same mutation in the CACNA1G gene was independently identified in the different ethnics, Japanese and French further supported the concept of CACNA1G as 
the causative gene for autosomal dominant SCA. Further studies are needed to clarify the clinical characteristics of the CACNA1G-dependent SCA in more detail.

In order to examine the role of CACNA1G in neurogenesis, iPSCs derived from the patient were differentiated into Purkinje cells. There were no significant defects in the morphology of Purkinje cells or the expression of Purkinje cell markers, and therefore, the mutation likely did not affect the differentiation of the cells into Purkinje cells. In the future, we are planning on conducting a functional evaluation of induced Purkinje cells.

\section{Conclusions}

In conclusion, we identified a mutation in CACNA1G encoding the T-type calcium channel from two families with dominantly inherited SCA. The mutation is located in the crucial residue of the S4 segment, which functions as a voltage sensor. Our electrophysiological study corroborated these changes in channel properties, and thus, the mutation was likely to be the cause of SCA. We indicated the novel evidence that ion channel dysfunction leads to the pathogenesis of SCA.

\section{Additional files}

Additional file 1: Figure S1. Brain MRI of the patient 2-III-1. The left panel shows the T1-weighted sagittal image, and the right panel shows the T1-weighted axial image. Marked cerebellar vermian atrophy was observed. (PPTX $1622 \mathrm{~kb}$ )

Additional file 2: Table S1. Summary of exome sequencing. (XLSX $42 \mathrm{~kb}$ ) Additional file 3: Table S2. Candidate variants. (XLSX $35 \mathrm{~kb}$ )

Additional file 4: Figure S2. Confocal images of HeLa cells expressing wild-type or p.Arg1715His mutant Cav3.1. HeLa cells were transiently transfected with wild-type or p.Arg1715His mutant human Cav3.1 and immunostained with anti- Cay3.1 antibodies (green) and anti-NaK-ATPase antibodies (red) as a membrane marker. Scale bar is $20 \mu \mathrm{m}$. (PPTX $286 \mathrm{~kb}$ )

Additional file 5: Figure S3. Morphological measurement of differentiated Purkinje cells. Dendritic field area (A) and diameter of cell soma (B) were measured in differentiated Purkinje cells. Data are presented as the mean \pm SD. Statistical significance was assessed by unpaired $t$-test. (PPTX $274 \mathrm{~kb}$ )

\section{Abbreviations \\ SCA: Spinocerebellar ataxia; EA: Episodic ataxia; gDNA: genomic DNA; SNP: Single nucleotide polymorphism; PCR: Polymerase chain reaction; iPSC: induced pluripotent stem cell; PBMC: Peripheral blood mononuclear cell; MRI: Magnetic resonance imaging; LOD: Logarithm of odds; VDCC: Voltage-dependent calcium channel; LTCP: Low-threshold calcium potential; NREM: Non-rapid eye movement.}

\section{Competing interest}

The authors report no disclosures relevant to the manuscript.

\section{Authors' contributions}

HK conceived and supervised this project. RM involved in Sanger sequencing. ROh and HMa provided advice on data analysis. TO and ROt involved in data acquisition. MW provided the CACNA1G antibody. HMo, YM, $\mathrm{KM}$, and $\mathrm{KH}$ analyzed the data and wrote the paper. All authors read and approved the final manuscript.

\section{Acknowledgments}

We thank Ms. Eiko Nakajima, Ms. Masayo Fujiwara, and Ms. Ayaka Nishiyama for their excellent technical assistance. This work was supported by a Grant-in-Aid for Scientific Research on Innovative Areas (Brain Environment) from the Ministry of Education, Culture, Sports, Science and Technology of Japan (H.K.), a Grant-in-aid for Scientific Research from the Japan Society for the Promotion of Science (H.Mo, 15 K15083; H.K., KAKENHI 26242085), and the Core Program for Disease Modeling Using iPS Cells from AMED (K.M.).

\section{Author details}

${ }^{1}$ Department of Epidemiology, Research Institute for Radiation Biology and Medicine, Hiroshima University, Hiroshima, 1-2-3, Kasumi, Minami-ku, Hiroshima 734-8553, Japan. ${ }^{2}$ Laboratory for Cell Asymmetry, RIKEN Center for Developmental Biology, Kobe, Japan. ${ }^{3}$ Department of Neurology, Tokyo Metropolitan Health and Medical Treatment Corporation Ebara Hospital, Tokyo, Japan. ${ }^{4}$ Clinical and Molecular Genetics, Hiroshima University Hospital, Hiroshima, Japan. ${ }^{5}$ Department of Anatomy, Hokkaido University Graduate School of Medicine, Sapporo, Japan. ${ }^{6}$ Department of Clinical Neuroscience \& Therapeutics, Graduate School of Biomedical and Health Sciences, Hiroshima University, Hiroshima, Japan. ${ }^{7}$ Department of Neurophysiology, Graduate School of Biomedical and Health Sciences, Hiroshima University, Hiroshima, Japan.

Received: 15 November 2015 Accepted: 21 December 2015 Published online: 29 December 2015

References

1. Durr A. Autosomal dominant cerebellar ataxias: polyglutamine expansions and beyond. Lancet Neurol. 2010;9:885-94.

2. Klockgether T. Update on degenerative ataxias. Curr Opin Neurol. 2011;24:339-45.

3. Zhuchenko O, Bailey J, Bonnen P, Ashizawa T, Stockton DW, Amos C, et al. Autosomal dominant cerebellar ataxia (SCA6) associated with small polyglutamine expansions in the alpha 1A-voltage-dependent calcium channel. Nat Genet. 1997;15:62-9.

4. Matsuyama Z, Kawakami H, Maruyama H, Izumi Y, Komure O, Udaka F, et al. Molecular features of the CAG repeats of spinocerebellar ataxia 6 (SCA6). Hum Mol Genet. 1997;6:1283-7.

5. Waters MF, Minassian NA, Stevanin G, Figueroa KP, Bannister JPA, Nolte D, et al. Mutations in voltage-gated potassium channel KCNC3 cause degenerative and developmental central nervous system phenotypes. Nat Genet. 2006;38:447-51.

6. Duarri A, Jezierska J, Fokkens M, Meijer M, Schelhaas HJ, den Dunnen WFA, et al. Mutations in potassium channel kcnd3 cause spinocerebellar ataxia type 19. Ann Neurol. 2012;72:870-80.

7. Lee Y-C, Durr A, Majczenko K, Huang Y-H, Liu Y-C, Lien C-C, et al. Mutations in KCND3 cause spinocerebellar ataxia type 22. Ann Neurol. 2012;72:859-69.

8. Jodice C, Mantuano E, Veneziano L, Trettel F, Sabbadini G, Calandriello L, et al. Episodic ataxia type 2 (EA2) and spinocerebellar ataxia type 6 (SCA6) due to CAG repeat expansion in the CACNA1A gene on chromosome 19p. Hum Mol Genet. 1997;6:1973-8.

9. Escayg A, De Waard M, Lee DD, Bichet D, Wolf P, Mayer T, et al. Coding and noncoding variation of the human calcium-channel beta4-subunit gene CACNB4 in patients with idiopathic generalized epilepsy and episodic ataxia. Am J Hum Genet. 2000;66:1531-9.

10. Sugihara K, Maruyama $H$, Morino $H$, Miyamoto $R$, Ueno $H$, Matsumoto $M$, et al. The clinical characteristics of spinocerebellar ataxia 36: a study of 2121 Japanese ataxia patients. Mov Disord. 2012;27:1158-63.

11. Gudbjartsson DF, Jonasson K, Frigge ML, Kong A. Allegro, a new computer program for multipoint linkage analysis. Nat Genet. 2000;25:12-3.

12. Morino H, Pierce SB, Matsuda $Y$, Walsh T, Ohsawa R, Newby M, et al. Mutations in Twinkle primase-helicase cause Perrault syndrome with neurologic features. Neurology. 2014;83:2054-61.

13. Li H, Durbin R. Fast and accurate short read alignment with Burrows-Wheeler transform. Bioinformatics. 2009;25:1754-60.

14. Li H, Handsaker B, Wysoker A, Fennell T, Ruan J, Homer N, et al. The Sequence Alignment/Map format and SAMtools. Bioinformatics. 2009;25:2078-9.

15. DePristo MA, Banks E, Poplin R, Garimella KV, Maguire JR, Hartl C, et al. A framework for variation discovery and genotyping using next-generation DNA sequencing data. Nat Genet. 2011;43:491-8. 
16. Wang K, Li M, Hakonarson H. ANNOVAR: functional annotation of genetic variants from high-throughput sequencing data. Nucleic Acids Res. 2010;38, e164.

17. Ramensky V, Bork P, Sunyaev S. Human non-synonymous SNPs: server and survey. Nucleic Acids Res. 2002;30:3894-900.

18. $\mathrm{Ng} \mathrm{PC}$, Henikoff S. Predicting deleterious amino acid substitutions. Genome Res. 2001;11:863-74.

19. Schwarz JM, Rödelsperger C, Schuelke M, Seelow D. MutationTaster evaluates disease-causing potential of sequence alterations. Nat Methods. 2010;7:575-6.

20. Hildebrand ME, Isope P, Miyazaki T, Nakaya T, Garcia E, Feltz A, et al. Functional coupling between mGluR1 and Cav3.1 T-type calcium channels contributes to parallel fiber-induced fast calcium signaling within Purkinje cell dendritic spines. J Neurosci. 2009;29:9668-82.

21. Okita K, Matsumura Y, Sato Y, Okada A, Morizane A, Okamoto S, et al. A more efficient method to generate integration-free human iPS cells. Nat Methods. 2011;8:409-12.

22. Okita K, Yamakawa T, Matsumura Y, Sato Y, Amano N, Watanabe A, et al. An efficient nonviral method to generate integration-free human-induced pluripotent stem cells from cord blood and peripheral blood cells. Stem Cells. 2013;31:458-66.

23. Muguruma K, Nishiyama A, Ono Y, Miyawaki H, Mizuhara E, Hori S, et al. Ontogeny-recapitulating generation and tissue integration of ES cell-derived Purkinje cells. Nat Neurosci. 2010;13:1171-80.

24. Muguruma K, Nishiyama A, Kawakami H, Hashimoto K, Sasai Y. Self-Organization of Polarized Cerebellar Tissue in 3D Culture of Human Pluripotent Stem Cells. Cell Rep. 2015;10:537-50.

25. Williams ME, Feldman DH, McCue AF, Brenner R, Velicelebi G, Ellis SB, et al. Structure and functional expression of alpha 1, alpha 2, and beta subunits of a novel human neuronal calcium channel subtype. Neuron. 1992;8:71-84.

26. Sigworth FJ. Structural biology: Life's transistors. Nature. 2003;423:21-2

27. Coutelier M, Blesneac I, Monteil A, Monin M-L, Ando K, Mundwiller E, Brusco A, Le Ber I, Anheim M, Castrioto A, Duyckaerts C, Brice A, Durr A, Lory P, Stevanin G. A Recurrent Mutation in CACNA1G Alters Cav3.1 T-Type Calcium-Channel Conduction and Causes Autosomal-Dominant Cerebellar Ataxia. Am J Hum Genet 2015;97:1-12.

28. Talley EM, Cribbs LL, Lee JH, Daud A, Perez-Reyes E, Bayliss DA. Differential distribution of three members of a gene family encoding low voltage-activated (T-type) calcium channels. J Neurosci. 1999:19:1895-911.

29. Tscherter A, David F, Ivanova T, Deleuze C, Renger JJ, Uebele VN, et al. Minimal alterations in T-type calcium channel gating markedly modify physiological firing dynamics. J Physiol. 2011;589(Pt 7):1707-24.

30. Park Y-G, Park H-Y, Lee CJ, Choi S, Jo S, Choi H, et al. Ca(V)3.1 is a tremor rhythm pacemaker in the inferior olive. Proc Natl Acad Sci U S A. 2010;107:10731-6

31. Lory P, Bidaud I, Chemin J. T-type calcium channels in differentiation and proliferation. Cell Calcium. 2006:40:135-46

32. Kim D, Song I, Keum S, Lee T, Jeong MJ, Kim SS, et al. Lack of the burst firing of thalamocortical relay neurons and resistance to absence seizures in mice lacking alpha(1G) T-type Ca(2+) channels. Neuron. 2001:31:35-45.

33. Ernst WL, Zhang Y, Yoo JW, Ernst SJ, Noebels JL. Genetic enhancement of thalamocortical network activity by elevating alpha $1 \mathrm{~g}$-mediated lowvoltage-activated calcium current induces pure absence epilepsy. J Neurosci. 2009;29:1615-25.

34. Lee J, Kim D, Shin H-S. Lack of delta waves and sleep disturbances during non-rapid eye movement sleep in mice lacking alpha1G-subunit of T-type calcium channels. Proc Natl Acad Sci U S A. 2004:101:18195-9.

35. Lampl I, Yarom Y. Subthreshold oscillations and resonant behavior: two manifestations of the same mechanism. Neuroscience. 1997:78:325-41.

\section{Submit your next manuscript to BioMed Central and we will help you at every step:}

- We accept pre-submission inquiries

- Our selector tool helps you to find the most relevant journal

- We provide round the clock customer support

- Convenient online submission

- Thorough peer review

- Inclusion in PubMed and all major indexing services

- Maximum visibility for your research

Submit your manuscript at www.biomedcentral.com/submit
C) Biomed Central 